\title{
Estilos de Crianza Parental en el Rendimiento Académico
}

\section{Parenting Styles on Academic Performance}

INFORMACIÓN DEL

\section{ARTÍCULO}

Fecha de recepción: 23 de Marzo de 2020.

Fecha de aceptación: 11 de Junio de 2020 .

\footnotetext{
Magíster en Orientación y Educación Familiar de la Universidad Técnica Particular de Loja. Docente e Investigadora de la Universidad Nacional de Loja.

E-mail: milena.vega@unl.edu.ec Código ORCID:

https://orcid.org/0000-0002-6845-9534
}

Milena Fernanda Vega Ojeda ${ }^{1}$

\section{Resumen}

Esta investigación permitió explorar la influencia de los estilos de crianza parental en el rendimiento académico, a través de la aplicación de la Escala de Afecto - Normas y Exigencias (Bersabé, Fuentes, y Motrico, 2001) a una muestra de 100 padres de familia y 100 estudiantes del noveno año de Educación Básica. Se identificó la forma como los hijos perciben a sus padres en cuanto a la educación que reciben, y la aplicación, cumplimiento de normas y aspectos inherentes a la convivencia familiar, mientras que los padres de familia evaluaron la forma como educan a sus hijos. El análisis realizado de los datos correspondientes a los estilos de crianza y los informes académicos de las calificaciones promedio de los estudiantes reveló que no hay relación aparente entre los rangos de calificaciones obtenidos por los estudiantes y sus estilos de crianza.

\section{Palabras Clave:}

Familia, estilos de crianza, escuela, rendimiento académico, normas, socialización, afecto.

Clasificación JEL: I21.

\begin{abstract}
This research allowed us to explore the influence of parenting styles on academic performance, through the application of the Scale of Affection-Norms and Requirements (Bersabé, Fuentes, y Motrico, 2001) to a sample of 100 parents and 100 students of the ninth year of Basic Education. The way in which children perceive their parents in terms of the education they receive was identified, as well as the application, compliance with norms and aspects inherent in family coexistence, while parents evaluated the way in which they educate their children.

The analysis of the data corresponding to the parenting styles and the academic reports of the average grades of the students revealed that there is no apparent relationship between the ranges of grades obtained by the students and their parenting styles.
\end{abstract}

CITACIÓN: Vega Ojeda, M.F. (2020) Estilos de Crianza Parental en el Rendimiento Académico. Podium, 37, 89-106. doi:10.31095/podium.2020.37. 7

\section{ENLACE DOI:}

http://dx.doi.org/10.31095/podium.202 0.37 .7

\section{Keywords:}

Family, parenting styles, school, academic performance, norms, socialization, affection.

JEL Classification: I21. 


\section{Introducción}

La familia constituye la primera instancia de socialización del ser humano, cuyo liderazgo lo asumen los padres, son quienes tienen la responsabilidad de educar, forma y guiar a sus hijos; sin embargo, en algunos casos, ésta acción es realizada usando los mismos patrones de crianza que recibieron en la infancia, o hacen lo contrario al estilo de crianza recibida que en ocasiones no es la adecuada. De ahí la importancia de que los padres presten mayor atención a sus prácticas parentales o estilos de crianza debido a que constituyen el eje en su rol educador, pues les brindarán herramientas para superar todo obstáculo que encuentre en su camino, de lo contrario se enfrentarán a un abismo interminable de dificultades donde los hijos son quienes asumen las consecuencias. Tan relevante es la influencia de la educación que reciben los hijos desde los primeros años de vida, que determina su accionar en ambientes diferentes al contexto familiar, uno de estos espacios, es la escuela, tan transcendental en la vida de los hijos, bien sea, porque es donde se encuentran la mayor parte de su tiempo o por ser el lugar donde se establecen las primeras interacciones entre sus pares. Y es así, que familia y escuela se encuentran estrechamente vinculadas influyéndose mutuamente; de tal manera que la importancia que dan los padres a la función educadora, la calidad de relaciones establecidas entre padres e hijos, y las estrategias que utilicen para educar, influyen notablemente en el rendimiento académico de los hijos. Uno de los estudios que ha relacionado los estilos de crianza parental y su influencia, específicamente en el rendimiento académico de los hijos, es el propuesto por Baumrind (1967), quien presenta dimensiones, como el apoyo y el control, y a partir de esta combinación da origen a diferentes estilos de crianza: autoritario, democrático y permisivo. Consecuentemente a través de sus estudios, demostró que las calificaciones de mayor puntaje son frecuentes en los hijos cuyos padres se caracterizaban por un estilo de crianza democrático, por el contrario, las bajas calificaciones son frecuentes en los hijos cuyos padres se caracterizan con un estilo negligente de crianza.

Estos hallazgos motivan la presente investigación, en la cual se empleó una metodología de tipo cuantitativa con un alcance de corte correlacional, debido a que busca identificar los estilos de crianza parental y su relación con el rendimiento académico. Se contó con una muestra de 100 estudiantes y 100 padres de familia, a quienes se les aplicó el instrumento Escala de Afecto-Normas y Exigencias de Bersabé, Fuentes, y Motrico (2001) en sus dos versiones para padres de familia e hijos. Se encontró que no hay una relación exacta respecto a que ante un estilo parental democrático exista un rendimiento académico satisfactorio. Los resultados demostraron que las calificaciones que oscilan entre 7 y 8 están presentes en los tres estilos de crianza estudiados, lo cual evidenció que los padres pueden manifestar diferentes estilos de crianza en la forma de comunicarse con sus hijos, y consecuentemente los efectos varían en el rendimiento 
académico.

\section{Revisión de literatura}

Los estilos de crianza engloban maneras, actitudes, y comportamientos, que generalmente utilizan los padres de familia en la tarea de educar a los hijos. $\mathrm{Su}$ relevancia ha incrementado por causa de su relación con los niveles emocional, afectivo y académico en los hijos, es así que se abordan temas como estilos de crianza (Baumrind, 1967) y efectos del estilo parental en el rendimiento académico.

\subsection{Estilos de crianza}

La familia es considerada como el primer lugar donde los padres educan y forman a los hijos, dirigiéndolos por el camino que creen correcto para su desarrollo, estableciendo niveles adecuados de afecto, comunicación y disciplina. En vista de que la forma de educar depende también de las características individuales de los padres e hijos, Darling y Steinberg (1993) afirman que:

El estilo de crianza, como una constelación de actitudes hacia el niño que se comunican al niño y que, en conjunto, crean un clima emocional en el que se expresan los comportamientos de los padres. Estos comportamientos incluyen tanto los comportamientos específicos dirigidos a objetivos a través de los cuales los padres desempeñan sus deberes parentales (para referirse a prácticas parentales) y comportamientos parentales no dirigidos a un objetivo, como gestos, cambios en el tono de voz o la expresión espontánea de emoción (p. 488).

La crianza parental depende de manera indudable de las características de la familia, fundamentalmente de los padres, donde los cambios vertiginosos en su estructura pueden alterar los roles de los integrantes y sus funciones, es así que todo aquello que transmitan, sean actitudes o normas, serán determinantes en su desarrollo. Sin embargo, la diversidad cultural y social, pone a los padres ante una difícil situación del uso de estrategias de crianza que anteriormente eran "normales" y que ahora tienen enfoques diferentes, en este sentido Moldes y Cangas (2011) señalan que:

Criamos a nuestros hijos bajo principios adquiridos durante nuestro propio desarrollo, transformados por opiniones culturales que subrayan qué es aceptable y qué es mejor. Las sociedades siempre han dictado las "reglas" de crianza buscando modelar el tipo de personas que se requieren en cada momento histórico (p.39).

De tal manera que la forma en que los padres emplean los estilos de crianza será responsable del desarrollo social y emocional de los hijos. "Desde muy temprano, los niños son criados bajo estilos particulares que orientan el desarrollo de sus creencias, conductas y valores" (Pereyra, Oros, Sicalo, y Robles, 2017, p. 120). Considerando que no siempre utilizarán cierto estilo educativo con todos, sin embargo, deben ser conscientes que como padres constituyen 
el soporte emocional, intelectual y social de los hijos en todo su ciclo de vida.

\subsubsection{Familia y crianza desde un} enfoque sistémico

La familia se considera como ente integrador, en el cual sus miembros inician el proceso de interacción dinámica que facilitará el funcionamiento de sus integrantes y las normas a cumplirse en el grupo familiar (Minuchin, 2009). La familia como sistema, se caracteriza porque está encabezada por las figuras paternas, las que están encargadas de cuidar el bienestar de cada uno de los integrantes y participar en el proceso de interacción con factores externos, lo cual es corroborado por Espinal, Gimeno, y González (2006), quienes afirman que:

A partir del enfoque sistémico los estudios de familia se basan, no tanto en los rasgos de personalidad de sus miembros, como características estables temporal y situacionalmente, sino más bien en el conocimiento de la familia como grupo con una identidad propia y como escenario en el que tiene lugar un amplio entramado de relaciones (p. 3).

En virtud de ello, cada miembro de la familia va configurando su identidad a través de las normas, límites e interacciones familiares, en donde su comportamiento afecta positiva, y en ocasiones, negativamente a todo el conglomerado familiar; al respecto se precisa que los padres ejerzan el papel de educadores basados en el diálogo y expresión de afecto, con la finalidad de crear y fortalecer vínculos familiares. Por lo tanto, en la familia cada miembro, sea padre, madre o hijos, tienen una función, cuyo cumplimiento aporta al mantenimiento de un sistema familiar. Ballenato (2007) manifiesta que:

Podemos definir un sistema como un conjunto de elementos que se interrelacionan entre sí, que se distribuyen determinadas funciones y las desarrollan, que se rigen por un conjunto de normas o reglas, manteniendo un cierto orden. Los sistemas se construyen con una finalidad, y una vez que alcanzan un cierto grado de estabilidad o equilibrio, tienden a mantenerlo (p. 46).

La búsqueda de la estabilidad familiar y emocional, conduce a los padres a adoptar una figura de equidad, donde a partir de la distribución de roles, considerando la edad de los miembros, dirigen, orientan, establecen límites y supervisan su cumplimiento, recordando que más que las palabras los hijos tendrán como ejemplo sus propias acciones. Por lo tanto, el contexto donde se desarrolla un grupo familiar es un factor relevante. "Aunque algunos padres estén sumamente preocupados por el desempeño de los hijos en las tareas escolares, deportivas y sociales, es conveniente señalarles que su función fundamental está en crear relaciones armoniosas y estrechas con ellos" (Valdés, 2007, p. 16). Efectivamente, la familia al ser un sistema abierto, necesita de padres que brinden orientación a sus integrantes sobre el proceso de establecer relaciones e interacciones con el medio, pudiendo ser una oportunidad a través de 
la participación en actividades lúdicas y familiares, en el tiempo que ellos dediquen a compartir con sus hijos.

\subsubsection{Crianza y proceso de socialización}

Los padres constituyen los primeros agentes de socialización para los hijos desde los primeros años de vida, se diferencia de otros tipos de socialización por los lazos de parentesco caracterizados por la existencia de fuertes niveles afectivos que se establecen entre padres e hijos. Por lo tanto, el proceso de socialización es una parte esencial en la crianza parental. Lo cual es corroborado por Martínez, Álvarez, y Fernández (2009):

De los aspectos educativos en los que está implicada la familia, la integración social de la persona, el desarrollo de habilidades para relacionarse e interactuar en el contexto social serían de los más determinantes para su futuro, pues este aprendizaje implica, entre otras cosas, la adquisición de la identidad, el logro de la autonomía y, en conjunto, el desarrollo de la capacidad de comunicación con los semejantes (p. 37).

Sin duda, la socialización desde la infancia, afianza la seguridad en los hijos, les brinda estabilidad emocional, y el desarrollo de una autoestima saludable, cabe mencionar que además depende de la forma de interacción entre los miembros de la familia. En consecuencia, la influencia de los padres y de la escuela sobre los hijos es trascendente en la socialización al momento de enseñar normas, valores y/o actitudes, que van modelando la personalidad del individuo, siendo la sociedad el escenario donde se manifestará como un ente social activo. "El proceso de socialización mediada por distintos agentes permite que el niño entre en relación con otros saberes, valores, costumbres y elementos materiales e ideales construidos $y$ acumulados por las anteriores generaciones" (Sánchez, 2001, p. 32). En tal razón, la familia liderada por los padres genera espacios de socialización como respuesta a los cambios en el entorno social, proporcionando a los hijos elementos nutricionales y de apoyo emocional para la apertura hacia contextos de tipo educativo, político, cultural, y religioso.

\subsubsection{Estilos de crianza según Baumrind}

Hay diversos trabajos de investigación sobre estilos de crianza parental, que hacen referencia a la forma de educar o criar a sus hijos por parte de los padres, y cómo se relaciona con el desarrollo emocional de los hijos, dada su relevancia en el proceso de socialización. En razón de ello, uno de los trabajos más elaborados y con mayor precisión, al definir los estilos de crianza parental, es el realizado por Baumrind citada en Torío, Peña, y Rodríguez (2008) quien realizó un estudio donde participaron 134 niños y niñas menores de 3 años escolarizados. Los datos fueron obtenidos a través de entrevistas a los padres y madres, y de la observación de la conducta de sus hijos, dando como 
resultado la siguiente clasificación de estructura personal de los niños:

- Estructura I: Competentes, contentos e independientes, confiados en sí mismos y mostraron conductas exploratorias.

- Estructura II: Retraídos, medianamente confiados, con poca tendencia a la aproximación $\mathrm{y}$, en cierto modo, inseguros y temerosos.

- Estructura III: Con bajo autocontrol y confianza, inmaduros y dependientes.

Y además, correlacionando características de la personalidad, obtuvo la siguiente clasificación de los padres de familia:

- Padres autoritativos: Se caracterizan por ser firmes en el cumplimiento de normas, y a la vez afectivos con sus hijos. Respetan la individualidad de cada integrante y su opinión en la toma de decisiones.

- Padres autoritarios: Se identifican por intentar controlar las conductas de sus hijos basados en normas que no pueden ser cuestionadas, y en caso de no ser acatadas por estos últimos, aplican castigos físicos.

- Padres permisivos: Se caracterizan por altos niveles de afecto, y por el contrario tienen bajo control en las malas conductas de sus hijos, debido a que generalmente existe ausencia en el cumplimiento de las insuficientes normas que aplican en el hogar.

Consecutivamente, con los niños que participaron en su primer estudio, cuando estos tenían 8-9 años de edad, observó que aquellos que correspondían a familias democráticas poseían competencias sociales y cognitivas elevadas; los que pertenecían a familias autoritarias se ubicaban en un nivel medio, y los niños de familias permisivas se situaban en los niveles más bajos. Finalmente, estudios llevados a cabo en años posteriores demostraron que, en la adolescencia, se mantenían los mismos efectos.

\subsubsection{Estilo democrático}

El estilo democrático se caracteriza porque está basado en los hijos, es decir, valoran y respetan la individualidad de los hijos, asimismo los padres establecen normas y reglas en el hogar considerando la opinión de los hijos en la toma de decisiones. "Es un estilo positivo, basado en la recompensa, los refuerzos, el reconocimiento y la alabanza, los padres se muestran dialogantes, cercanos. Establecen una comunicación bidireccional con los hijos, en los que prima la escucha" (Ballenato, 2007, p.108). Es decir, la comunicación tiene un valor fundamental como recurso de negociación, expresión de sentimientos y resolución de conflictos, además promueve el desarrollo de habilidades sociales que servirán para establecer relaciones inter e intrapersonales saludables.

Los altos niveles de afecto están de igual manera relacionados con los niveles de exigencia que los padres de familia emplean para el cumplimiento de mandatos (Gaspar, 2016). Los padres son conscientes de que sus acciones son imitadas por sus hijos, por lo que en lo posible actúan de manera coherente a lo que demandan. 


\subsubsection{Estilo autoritario}

El estilo de crianza autoritario "es un modelo coercitivo, represivo, dictatorial, que está basado en la imposición, la severidad y el sometimiento. Se intenta imponer por la fuerza una autoridad de los padres que se presenta como incuestionable" (Ballenato, 2007, p.107). Es decir, que está centrado en los padres, con una comunicación unidireccional, misma que se manifiesta en una actitud desafiante que intimida y sobrevalora la opinión de sus hijos, imponen normas sin considerar opinión alguna por parte de los hijos, y sin embargo, exigen su cumplimiento de lo contrario utilizan el castigo como recurso según su criterio, para educar.

En ocasiones, los padres usan la violencia física como recurso para imponer su autoridad, acciones que son frecuentes cuando los hijos son pequeños, no obstante, este panorama puede ser distinto en hijos mayores, en consecuencia, los hijos poseen una baja autoestima como resultado de no sentirse aceptados y respetados, lo cual podría desencadenar en un desajuste social y emocional.

\subsubsection{Estilo Permisivo}

El estilo permisivo se caracteriza porque prevalece la opinión e interés del hijo sobre el rol parental que deben asumir los padres, pero que en ocasiones la crianza es delegada a otras personas., por lo tanto, la ausencia de límites facilita al hijo actuar sin control, haciendo lo que le gusta cuando él quiera, y cuando no se cumplen sus deseos se observa comportamientos agresivos, los cuales no son frecuentes porque normalmente los padres terminan cumpliendo sus exigencias. Lo cual es ratificado por Ballenato (2007) quien afirma:

El modelo muestra a unos padres que no se implican afectivamente, que se mantienen al margen. Da la impresión de que han tirado la toalla y han renunciado a su labor educativa, se manifiestan insatisfechos con la conducta de sus hijos, pero no hacen nada para cambiar la situación ( $p$. 109).

De tal manera, que los padres con este estilo de crianza, asumen una actitud de conformismo ante las innumerables conductas inadecuadas de sus hijos, lo que, a su vez, sin darse cuenta, promueven en los hijos la falta de cumplimiento de actividades, tareas escolares o familiares, y a la larga un comportamiento libertino.

\subsubsection{Efectos del estilo parental sobre el rendimiento académico}

Las prácticas educativas son empleadas por los padres en su labor de educar y criar a los hijos, sin embargo, algunas de estas prácticas no son apropiadas en la relación padre-hijo, algunas dependen de características individuales de los padres como su personalidad, factores sociales $\mathrm{y}$ culturales $\mathrm{y}$, como eje predictor, el valor que dan los padres a la educación $\mathrm{y}$ formación de sus hijos en su preparación para la vida. Por otra parte, el estudio realizado por Aunola, Stattin y Nurmi 
(citados en Raya, 2009), analizaron las estrategias de logro académico empleadas por los estudiantes y su relación con el estilo educativo de sus padres, quienes concluyeron lo siguiente:

- Familias democráticas: Aplicaban las estrategias más adaptativas en situaciones académicas, y tenían bajos niveles de expectativas de fracaso, conductas poco útiles, pasividad; y un alto nivel de autoestima.

- Familias permisivas: Mostraban las habilidades menos adaptadas, con altos niveles de pasividad y conductas poco útiles y baja autoestima.

- Familias autoritarias: Revelaban actitudes similares a los de las familias negligentes en cuanto a sus estrategias de aprendizaje. Estrecha relación entre los hijos de familias permisivas $y$ autoritativas.

Se evidencia que los padres que educan basados en una comunicación activa con sus hijos y brindan confianza para expresar sentimientos, originan cualidades positivas que favorecen el desarrollo de la autonomía y autoestima, por el contrario el escaso diálogo entre padres e hijos y excesiva exigencia en el cumplimiento de normas sin considerar la opinión de los hijos, además la ausencia de expresiones afecto, desencadena en baja autoestima, problemas de autocontrol y rendimiento académico no satisfactorio.

\subsection{Rendimiento Académico}

Los estilos de crianza son un factor relevante en el proceso de enseñanza- aprendizaje, debido a la relación directa con el rendimiento académico de los hijos. Existe una multiplicidad de factores alrededor de esta relación que determinan un rendimiento académico satisfactorio, tales como: Nivel intelectual, interés por el estudio, personalidad, habilidades, motivación académica, pero con mayor notabilidad es la influencia del entorno familiar, la aceptación, control de los padres, y el afecto quienes viabilizan a los hijos para alcanzar una alta competencia académica.

El rendimiento académico es en sí la derivación de las sapiencias aprendidas por el estudiante como parte de su preparación académica, y su capacidad de respuesta a diferentes elementos que inciden en su formación (Molina, 2015). Posteriormente, a partir de recursos empleados por el docente como lecciones, tareas o entrevistas, evalúa el nivel de conocimientos alcanzados y otorga una calificación de tipo cuantitativa, en este sentido tanto docente como estudiante son elementos trascendentales en el proceso educativo, cuyo escenario es el salón de clase.

Partiendo de los supuestos anteriores, el rendimiento académico es el logro máximo del alumno como resultado de su participación en el proceso de enseñanza-aprendizaje en la institución educativa a la que pertenezca (Martínez-Otero, 1997), considerado una dimensión multifactorial, donde convergen dimensiones como la motivación académica, interés, dedicación, además de factores ligados al ambiente familiar. Por ello, la comunidad educativa 
conformada por directivos, docentes, estudiantes y padres de familia en los contextos familiar y educativo, como acción preventiva sepan identificar oportunamente posibles conflictos que puedan afectar el desenvolvimiento académico del estudiante.

\subsubsection{Relación familia y escuela}

En la familia, el hijo aprende desde la infancia a comunicarse y relacionarse con su entorno inmediato que son sus padres y hermanos, quienes sirven de modelo de conductas, creencias y valores; los padres garantizan cuidar de sus necesidades básicas, asegurar el bienestar emocional y desarrollo psicológico, socializar normas, inculcar valores y desarrollar hijos autónomos e independientes. La familia establece una alianza con los docentes desde inicios de la vida escolar de los hijos, significante apoyo en el proceso de aprendizaje. Martínez-Otero afirma:

La importancia del profesor en todo el proceso educativo es un hecho innegable que en la actualidad nadie cuestiona. Es evidente, sin embargo, que el papel que los docentes ha jugado en la sociedad no ha sido siempre el mismo, como tampoco ha sido siempre igual la imagen que se ha tenido de los enseñantes (p. 32).

De tal manera que la familia al ser la primera escuela para los hijos, es también el contexto que catapulta al éxito o fracaso y que sirve de base para aprendizajes posteriores en la vida de los hijos.
1.2.2 Participación de los padres de familia en el proceso de aprendizaje de los hijos

La familia y la escuela son dos escenarios que dependen entre sí, dos vías principales a través de las cuales los hijos adquieren aprendizajes, desarrollan su personalidad y progresivamente se van convirtiendo en adultos con autoestima, autonomía, y seguridad, preparados para afrontar diversas situaciones a lo largo de su vida. Dentro de este marco, los docentes han dejado de lado transmitir conocimientos para pasar a educar en normas, límites y valores. Bolívar (2006) afirma:

En los últimos tiempos, los profesores se quejan, con razón, de cómo ante determinadas situaciones conflictivas, la actitud más común de los padres es la de apoyar a sus hijos, en vez de colaborar. Es preciso romper las fronteras de territorios separados, cuando de lo que se trata es del objetivo común de educación para la ciudadanía (p. 133).

No cabe duda, que esta situación refleja de manera preocupante la ausencia de vínculos entre dos contextos, que por el contrario deberían estar fortalecidos para hacer frente a situaciones cotidianas complejas, a través de una educación y orientación oportuna. Si bien es cierto la familia es el primer lugar de socialización para los hijos, no es el único, existen múltiples caminos e individuos que trasladan diversidad de información hasta ellos. Al respecto Bolívar (2006) menciona: "Ni la escuela es el único contexto de educación, ni sus profesores 
y profesoras los únicos agentes, al menos también la familia y los medios de comunicación desempeñan un importante papel educativo" (p.120). De allí que la orientación y enseñanza por parte de los padres sobre lo que ven y escuchan en los medios digitales, se considera como una acción preventiva, ante información con contenido no formativo.

\subsubsection{Factores que influyen en el Rendimiento Académico}

Con el objeto de delimitar los factores que intervienen en el rendimiento académico, se toma como referencia el estudio realizado por Montero y Villalobos (2007), quienes agrupan cuatro grandes factores: Institucional, pedagógico, psicosocial y sociodemográfico, los cuales describen a continuación:

1.2.3.1 Factores Institucionales: Son aquellos elementos externos que al interactuar con el estudiante ejerce alta influencia en el rendimiento académico, tales como: Metodología, número de alumnos, horario, los tamaños de los grupos, número de libros en la biblioteca del centro educativo, aspectos relacionados con la carrera que sigue el (la) estudiante y el ambiente institucional (Montero y Villalobos, 2007). Al respecto, existen aspectos que se pueden manejar en el contexto educativo como modificar un horario o reducir el número de estudiantes por grupo escolar.

1.2.3.2 Factores Pedagógicos: En este componente tiene como base la función del docente, las diferentes tácticas de enseñanza utilizadas, los métodos de evaluación y materiales didácticos (Montero y Villalobos, 2007). A más de la motivación que reciben los estudiantes y el periodo que dedica en la preparación de sus clases y actividades escolares a desarrollar con el estudiantado.

1.2.3.3 Factores psicosociales: Page (citado en Montero y Villalobos, 2007) hace hincapié a varios elementos que forman parte de la personalidad del estudiante como su autoestima, seguridad, estado emocional, y su visión sobre el estudio, la cual además está influenciada por la importancia proyectada por el/la docente en el proceso de aprendizaje.

En este sentido, la dedicación que el estudiante aporta en sus actividades escolares utilizando sus habilidades y actitudes le permite apreciar sus logros académicos sintiéndose orgulloso de su esfuerzo.

1.2.3.4 Factores sociodemográficos: Se refiere a elementos externos cuyas características pertenecen a la familia, tales como: Lugar donde viven por la relación con el componente cultural, nivel de preparación académica de los padres, nivel socioeconómico debido a que se considera como un agente causal del éxito o fracaso en el área académica, tipo de centro educativo donde cursa los estudios o donde los ha culminado (Montero y Villalobos, 2007), y por último, un elemento relevante el sexo del estudiante, ya que culturalmente se considera relegada la posición de la mujer, subvalorando su inteligencia y capacidad de alcanzar un buen 
rendimiento académico.

\subsubsection{Indicador del Rendimiento Académico}

El rendimiento académico señala el nivel de conocimientos adquiridos en las asignaturas que forman parte del currículo académico. Latiesa (como se citó en Garbanzo, 2007) afirma:

El resultado de la relación entre lo que el estudiante aprendió y lo que alcanza en los instrumentos de valoración utilizados por el docente para conocer su nivel de conocimientos, se refleja en la calificación que obtiene, y que, corresponde a un determinado periodo académico

Los resultados se obtienen a partir de las valoraciones realizadas a los estudiantes, de las que se obtiene un puntaje que directamente tiene concordancia con el nivel de conocimiento. Los recursos que generalmente se utilizan para medir los resultados obtenidos de evaluaciones, son las calificaciones escolares, en virtud de lo mencionado son el único criterio social y legal que otorga un valor al nivel de conocimientos alcanzados en los estudiantes (Solano, 2015).

\section{Metodología}

La presente investigación es de tipo cuantitativa y tiene un alcance descriptivo, debido a que busca identificar los estilos de crianza parental, y el nivel de rendimiento en los estudiantes del noveno año de Educación Básica. Se emplearon dos escalas: La de afecto (EA) y la de
Normas y Exigencias (ENE) de Bersabé, Fuentes, y Motrico (2001). La EA se compone de dos factores: 1) Afectocomunicación y 2) Crítica-rechazo de los padres hacia sus hijos. Cada uno de los factores consta de 10 ítems que se contestan en una escala tipo Likert con 5 grados de frecuencia (nunca, pocas veces, algunas veces, a menudo y siempre). La puntuación total de cada factor está comprendida entre 10 y 50 . La ENE consta de 3 factores: 1) Forma inductiva, 2) Forma rígida y 3) Forma indulgente que tienen los padres de establecer y exigir el cumplimiento de las normas. Los dos primeros factores tienen 10 ítems, y el tercero se compone de 8. La escala de respuesta es la misma que en la EA, por lo que la puntuación total de los dos primeros factores también está comprendida entre 10 y 50 . Únicamente la puntuación del tercer factor varía entre 8 y 40.

Tabla 1.

Estudiantes del noveno año de Educación Básica

\begin{tabular}{cc}
\hline PARALELO & $\begin{array}{c}\text { NÚMERO DE } \\
\text { ESTUDIANTES }\end{array}$ \\
\hline A & 32 \\
B & 30 \\
C & 29 \\
F & 34 \\
TOTAL & $\mathbf{1 2 5}$ \\
\hline
\end{tabular}

Fuente: Unidad Educativa Fiscomisional "San Francisco de Asís".

En la Tabla 1 se presenta la distribución por paralelos de la población de estudiantes de noveno año de educación básica de la Unidad Educativa 
Fiscomisional "San Francisco de Asís". Para el cálculo del tamaño de muestra se utilizó un nivel de confianza del 95\% y un error del 3\%, requiriéndose incluir en la investigación un total de 100 estudiantes, y por consiguiente 100 padres de familia.

El procedimiento de recolección de datos consistió en lo siguiente:

1) Se realizó un acercamiento al Director Distrital 19D01 Educación y a la Rectora de la Institución Educativa, a quienes se entregó un oficio para solicitar la autorización y colaboración en la realización del trabajo de investigación.

2) Al contar con la respectiva autorización por parte de las autoridades educativas, se contó con el apoyo y colaboración de Inspectores, Docentes, y Tutores de la Institución Educativa, quienes conocieron del tema e instrumentos a aplicar en el trabajo investigativo.

3) Se procedió a la aplicación de la Escala de Afecto, Normas-Exigencias a los estudiantes de los Novenos Años de Educación Básica (previa selección de la muestra).

4) Aplicación de la Escala de Afecto y de Normas-Exigencias (versión padres), a los padres de familia.

5) Se recopilaron los cuestionarios de los padres de familia y de los estudiantes, y fueron analizados.

6) Previa autorización de la Autoridad de la Institución Educativa y padres de familia participantes de la investigación, se entregaron a la autora de la investigación los informes académicos de las calificaciones promedio de los estudiantes, cuyos datos se utilizaron para medir el rendimiento académico.

7) Finalmente, la codificación de datos se realizó mediante el uso del programa estadístico SPSS, cuyos resultados están expresados en formato numérico. La información adquirida se representó a través de tablas, gráficas, análisis e interpretaciones.

\section{Resultados}

Los estilos de crianza guardan estrecha relación con el rendimiento académico, y en una etapa como la adolescencia conocida por los múltiples cambios que atraviesan propios del desarrollo, aspectos como la comunicación, el afecto, las normas establecidas en consenso entre padres e hijos, la participación en conjunto en actividades lúdicas, representan la importancia de la influencia de los estilos de crianza, la implicación de los padres de familia constituyen en su mayoría el determinante para alcanzar altas puntuaciones en el proceso de estudio.

Estos resultados son el producto de la aplicación de la Escala de Afecto, Normas y Exigencias en sus dos versiones (padres e hijos), cuyos resultados se analizaron y correlacionaron con los informes académicos de las calificaciones promedio de los estudiantes (sujetos de investigación). 


\section{Resultados cuestionario (versión hijos)}

La Figura 1, representa de manera general los valores obtenidos, a partir del análisis comparativo entre ambos factores que componen la Escala de Afecto (EA). Considerando que la puntuación fluctúa entre 10 la mínima y 50 la máxima, los resultados obtenidos en el factor CríticaRechazo se concentra principalmente en el rango 21-30 representado por un $77 \%$. En cambio, en el factor AfectoComunicación, los valores más altos corresponden a los rangos 31-40 y 41-50, alcanzando un total de $80 \%$.

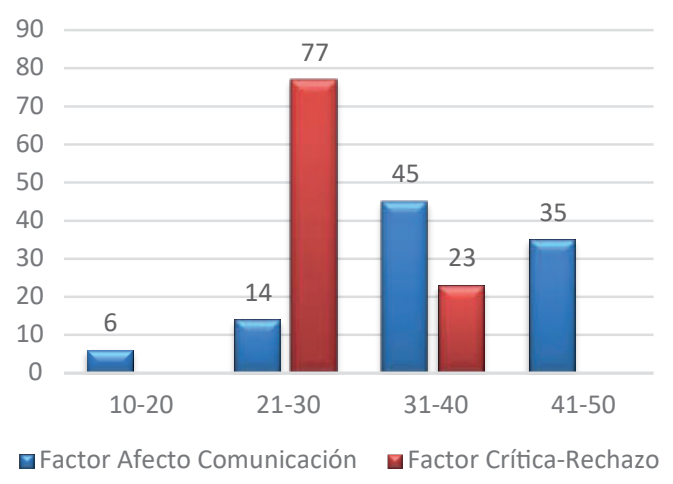

Figura 1. Escala comparativa Afecto-Crítica (versión hijos). Fuente: Base de datos de estudiantes.

La Figura 2, representa los resultados correspondientes a la evaluación realizada por los hijos a la forma de crianza de los padres. El rango 41-50 corresponde a las puntuaciones más altas, lo representa el estilo inductivo o también denominado democrático, alcanzando un $44 \%$, siendo una puntuación alta en relación al 23\% que pertenece a la forma rígida. El segundo rango que fluctúa entre 31-40, sus valores alcanzan el $45 \%$ para la forma inductiva y el $47 \%$ para la forma rígida. Obteniéndose un valor de $89 \%$ para la forma inductiva y $70 \%$ para la forma rígida.

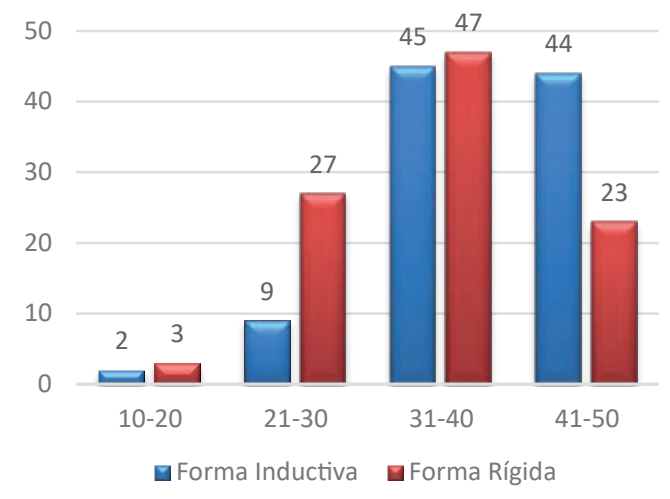

Figura 2. Escala de Normas y Exigencias Inductiva y Rígida (versión hijos).

Fuente: Base de datos de estudiantes.

La Figura 3, representa al estilo parental indulgente o permisivo, el rango comprendido entre 8-18 cuyas puntuaciones son las más bajas de entre los factores, se encuentra representado por el $79 \%$.

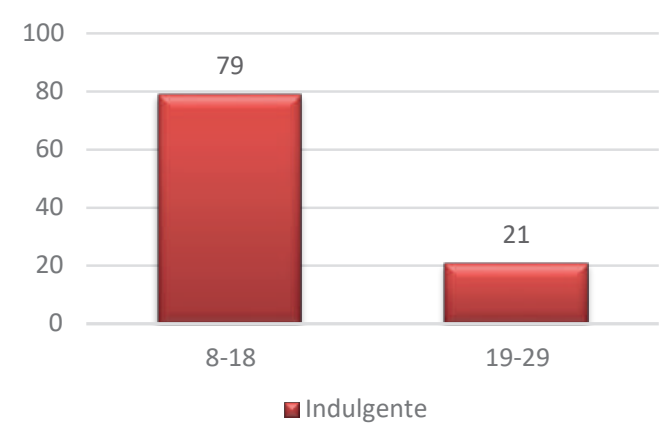

Figura 3. Escala de Normas y Exigencias Indulgente (versión hijos).

Fuente: Base de datos de estudiantes.

\section{Resultados cuestionario (versión padres)}

Los cuestionarios de Escala-Afecto y Escala de Normas y Exigencias también fueron aplicados a los padres, esto con la finalidad de contrastar los resultados obtenidos. Del total de instrumentos contestados el $76 \%$ fueron respondidos por la madre, el $21 \%$ por el padre, y el $3 \%$ por una tía o tío. Con respecto al estado civil de los padres, el $54 \%$ están casados, $22 \%$ 
tienen unión libre y $18 \%$ son solteros. A partir de la metodología expuesta, se presentan los resultados del análisis psicométrico de la Escala de Afecto en la versión que contestan los padres refiriéndose a cómo es su conducta concreta con su hijo/a.

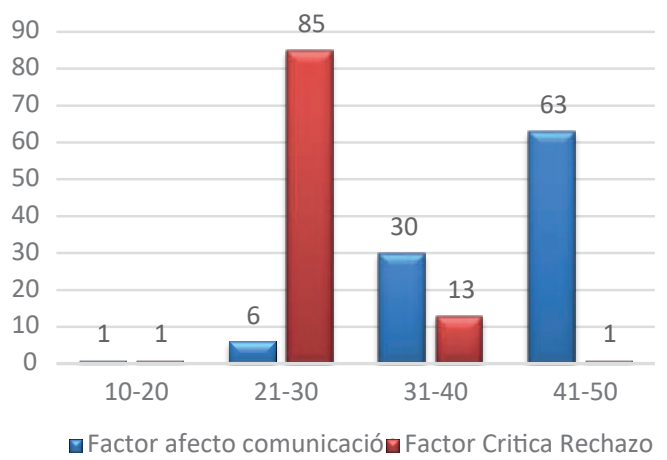

Figura 4. Escala comparativa Afecto-Crítica (versión padres). Fuente: Base de datos de padres.

La Figura 4, representa los valores obtenidos de la escala de Afecto Comunicación y Crítica-Rechazo aplicada a los padres, siendo 10 la mínima y 50 la máxima. Se puede evidenciar que el mayor porcentaje se concentra en el rango de 21 a 30 con un $85 \%$ para el factor Crítica-Rechazo, mientras que en el rango de 41 a 50 está representado por el $63 \%$ para el factor AfectoComunicación. De tal manera, el factor Afecto-Comunicación alcanza un 93\%, y el factor Crítica-Rechazo el 14\%.

La Figura 5 representa los valores obtenidos, a partir del análisis comparativo entre ambos factores que componen la Escala de Normas y Exigencias (ENE). Según los resultados obtenidos, la forma inductiva se encuentra entre 31 y 50 obteniéndose un resultado total de $91 \%$, en cambio en la forma rígida se obtuvo un total del $89 \%$.
Estos hallazgos de la forma inductiva coinciden con lo afirmado por Ballenato (2007): "Es un estilo positivo, basado en la recompensa, los refuerzos, el reconocimiento y la alabanza, los padres se muestran dialogantes, cercanos. Establecen una comunicación bidireccional con los hijos, en los que prima la escucha" (p.108). Lo que permite a los padres dar una imagen asertiva y segura, donde los hijos aprenden el valor del respeto hacia ellos, y a sí mismos, además de sentirse aceptados y respetados, en consecuencia, el estilo inductivo contribuye al desarrollo emocional en los hijos.

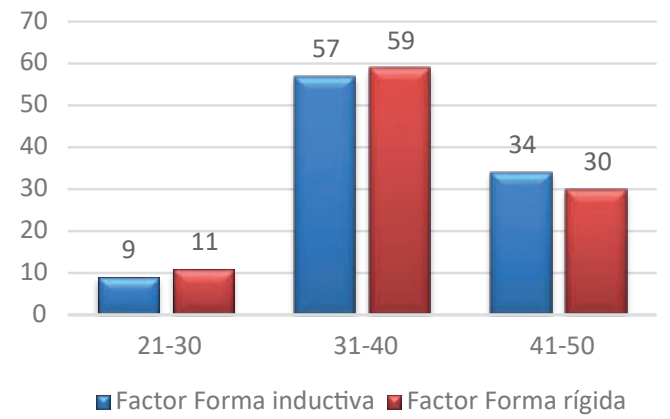

Figura 5. Escala de Normas y Exigencias Inductiva y Rígida (versión padres).

Fuente: Base de datos de padres.

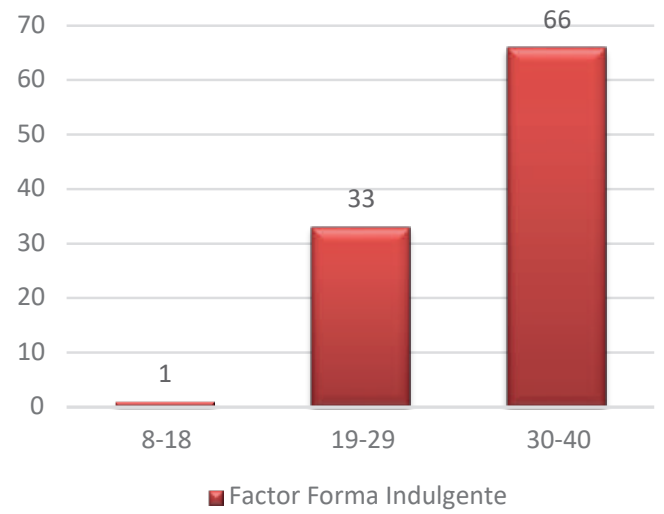

Figura 6. Escala de Normas y Exigencias Indulgente (versión padres).

Fuente: Base de datos de padres.

La Figura 6 muestra al factor 
indulgente en el rango 30-40 con el 66\%. Los resultados evidencian que los padres se caracterizan por un estilo de crianza indulgente, son aquellos que no ponen normas ni límites a la conducta de sus hijos y, si lo hacen, no exigen en su totalidad su cumplimiento. Lo cual se corrobora, con lo expresado por Ballenato (2007) quien afirma:

El modelo muestra a unos padres que no se implican afectivamente, que se mantienen al margen. Da la impresión de que ha tirado la toalla y han renunciado a su labor educativa, se manifiestan insatisfechos con la conducta de sus hijos, pero no hacen nada para cambiar la situación ( $\mathrm{p}$. 109).

Es importante que los padres establezcan límites, normas y supervisen que se cumpla cuando los hijos son aún pequeños, de lo contrario en la adolescencia pueden entrar en conflicto ante la ausencia de control, o bien aún demostrar dependencia hacia los padres, dando lugar al incumplimiento de responsabilidades familiares y escolares.

Los estilos de crianza parental y el rendimiento académico

Como se observa en la Figura 7, no existe una relación clara de que mientras mayor sea el factor Forma Inductiva, mayores sean las calificaciones de los estudiantes. El mayor número de estudiantes (42) se concentra en el rango intermedio de 31-40, con notas comprendidas entre 7,01 y 9. Los resultados corroboran lo que señala Bolívar (2006): "Ni la escuela es el único contexto de educación, ni sus profesores y profesoras los únicos agentes, al menos también la familia y los medios de comunicación desempeñan un importante papel educativo" (p.120). De tal manera, que existen otros factores que inciden para que los hijos obtengan un rendimiento académico satisfactorio, por ello los padres necesitan utilizar estrategias o pautas que deben establecerse de manera clara en el hogar en cuanto al uso de los medios tecnológicos, pueden ser un importante recurso si los hijos cuentan con la supervisión de los padres, sino de lo contrario tendrán acceso a contenido no formativo contrario a una educación parental basada en valores.

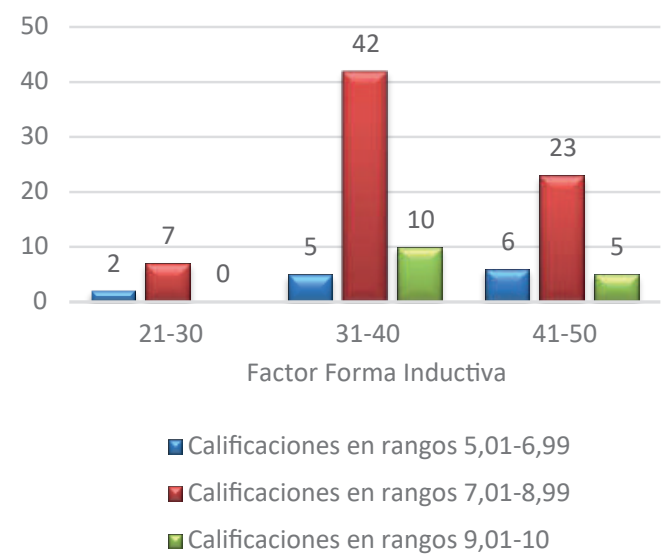

Figura 7. Resultados Escala Normas y Exigencias Inductiva -Calificaciones.

Fuente: Base de datos de padres y estudiantes.

Como se puede observar en la Figura 8 , el mayor número de estudiantes se concentra en el rango de 31-40 (44), el cual es un rango intermedio que comprende notas entre 7,01 y 9; únicamente 7 estudiantes poseen calificaciones entre 9,01 y 10 en el rango de 41-50. Valdés (2007) afirma: "Aunque algunos padres estén sumamente 


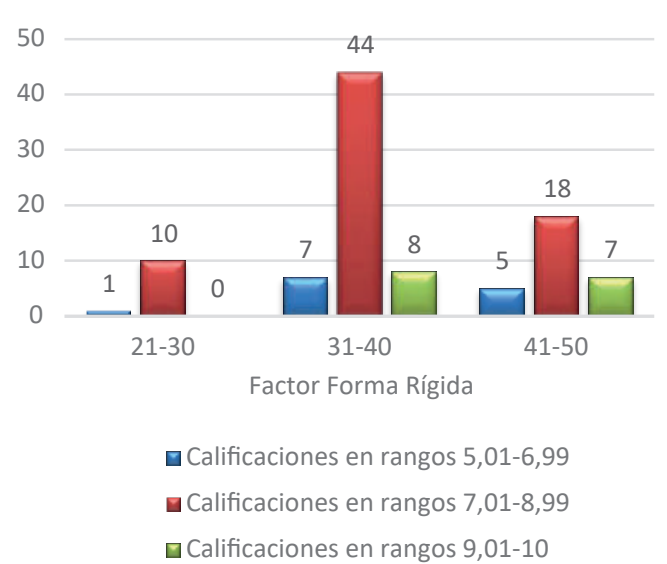

Figura 8. Resultados Escala Normas y Exigencias RígidaCalificaciones.

Fuente: Base de datos de padres y estudiantes.

preocupados por el desempeño de los hijos en las tareas escolares, deportivas y sociales, es conveniente señalarles que su función fundamental está en crear relaciones armoniosas y estrechas con ellos" (p. 16). De tal manera que se evidencia que los padres pueden manifestar diferentes estilos de crianza, sin embargo, dejar de lado el diálogo, el afecto y la promulgación de valores como el respeto, sin duda tendrá efectos poco favorables en el proceso de estudio.

En la Figura 8, se observa que los resultados obtenidos no guardan relación con las calificaciones alcanzadas por los estudiantes. El mayor número de estudiantes (44) se concentra en el rango de 30-40, con notas entre 7,01 y 9 .

\section{Conclusiones}

En el análisis de resultados en la Escala de Normas y Exigencias, se comprueba que un número significante de padres de familia refieren tener un estilo de crianza democrático, el mismo

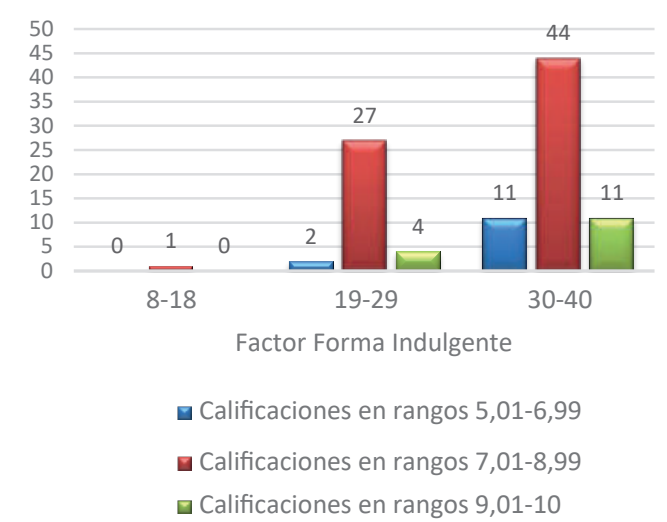

Figura 9. Resultados Escala Normas y Exigencias Indulgente-Calificaciones.

Fuente: Base de datos de padres y estudiantes.

que da lugar a una comunicación de calidad entre padres e hijos, seguido por un estilo autoritario en el que las normas se imponen sin consenso y una disciplina demasiado estricta, y por último el estilo parental permisivo que hace referencia a las escasas reglas y exigencia al momento de establecerlas y hacerlas cumplir, independientemente del estilo parental, para los hijos la familia se considera como una importante red de apoyo y ejerce una significativa influencia en sus actitudes, valores y creencias, que se reflejan en su rendimiento académico.

En cuanto a los hallazgos de la aplicación de la Escala de AfectoComunicación versión padres, que incluye entre otros elementos la valoración auténtica del hijo, el interés, el apoyo emocional y la comunicación, obtuvo un $93 \%$ entre los rangos con mayor puntuación, guardando una estrecha relación con los resultados alcanzados en la Escala de Normas y Exigencias donde queda demostrado que aspectos como el afecto y la 
comunicación propios del estilo parental democrático influyen positivamente en el rendimiento académico; en cambio el estilo parental con alto nivel de crítica y rigidez en el acatamiento de reglas limitaría a los hijos en conseguir un rendimiento académico satisfactorio.

Es así que el estilo democrático de crianza es predominante entre los padres de familia, caracterizado por las expresiones de afecto, interés y compromiso con la educación de los hijos, en razón de aquello obedece que en su gran mayoría las calificaciones obtenidas por los estudiantes se encuentren entre los rangos medios, lo que demuestra que aquellos hijos tienen una visión positiva hacia su preparación académica, lo que supone un elemento transcendental en la consecución de sus objetivos escolares. No obstante es preocupante los datos obtenidos en el Factor Crítica-Rechazo versión hijos, que alcanzó un $77 \%$, lo cual se evidencia en las expresiones de los adolescentes quienes sienten que sus derechos son quebrantados, no se sienten aceptados ni respetados, manifestándose de manera significativa en su autoconcepto académico y en sus calificaciones que no alcanzaron los niveles más altos o muy satisfactorios, de igual forma existe relación con el estilo parental permisivo que se encuentra por debajo del estilo autoritario y democrático.

Adicionalmente, los datos obtenidos entre los estilos de crianza estudiados y los informes académicos de las calificaciones promedio de los estudiantes revelaron que no hay relación aparente entre el estilo democrático y altas calificaciones; las calificaciones entre 7,01 y 9 predominaron en los tres estilos de crianza, lo cual evidenció que los padres pueden manifestar diferentes estilos de crianza en la forma de comunicarse con sus hijos. Además, se debe tener en cuenta que los adolescentes están bajo la influencia de otros factores como los medios de comunicación, el uso de la tecnología, grupos sociales, etc. en razón de ello únicamente los estilos de crianza no influirían en el rendimiento académico.

Los padres de familia son elementos imprescindibles en el desarrollo de los hijos, su involucramiento en la crianza es fundamental para su educación, por lo que se propone en coordinación con el Departamento de Consejería Estudiantil (DECE) y la autoridad de la Institución Educativa, socialicen los resultados de los estilos de crianza parental democrático, autoritario, y permisivo identificados en los padres de familia, y se brinde la orientación oportuna, con la finalidad de augurar un mayor compromiso y responsabilidad sobre la forma de educar y guiar en la vida familiar, y en el proceso educativo de sus representados; conjuntamente es relevante que se establezcan de manera periódica jornadas de reflexión o convivencias, que cuenten con la participación de padres e hijos, como espacios de encuentro ante la necesidad existente del diálogo, afecto, y aceptación en las relaciones paternofiliales. 


\section{Referencias}

Ballenato, G. (2007). Educar sin gritar. Padres e hijos: ¿convivencia o supervivencia? Madrid: La Esfera de los Libros.

Baumrind, D. (1967). Child care practices anteceding three patterns of preschool behavior. Genetic Psychology Monographs, 75(1), 43-88.

Bersabé, R., Fuentes, M. J., y Motrico, E. (2001). Análisis psicométrico de dos escalas para evaluar estilos educativos parentales. Psicothema, 13(4), 678-684.

Bolívar, A. (2006). Familia y Escuela: dos mundos llamados a trabajar en común. Revista de Educación, (339), 119-146.

Darling, N., y Steinberg, L. (1993). Parenting style as context: An integrative model. Psychological Bulletin, 113(3), 487-497.

Espinal, I., Gimeno, A., y González, F. (2006). El enfoque sistémico en los estudios sobre la familia. Revista Internacional de Sistemas, 14, 1-14.

Garbanzo, G. (2007). Factores asociados al rendimiento académico en estudiantes universitarios, una reflexión desde la calidad de la educación superior pública. Educación, 31(1), 43-63.

Gaspar, A. (2016). Familia, Sicoética y Valores. Loja: EDILOJA.

Martínez-Otero, V. (1997). Los adolescentes ante el estudio: Causas y consecuencias del rendimiento académico. Madrid: Editorial Fundamentos.

Martínez, M., Álvarez, B., y Fernández, A. (2009). Orientación Familiar: contextos, evaluación e intervención. Madrid: Sanz y Torres.

Minuchin, S. (2009). Familias y terapia familiar. Barcelona: Editorial Gedisa.
Moldes, P., y Cangas, A. J. (2011). La comunicación entre padres e hijos. Málaga: Editorial Arguval.

Molina, M. L. (2015). Valoración de los criterios referentes al rendimiento académico y variables que lo puedan afectar. Revista Médica Electrónica, 37(6), 617-626.

Montero, E., y Villalobos, J. (2007). Factores institucionales, pedagógicos, psicosociales y sociodemográficos asociados al rendimiento académico en la Universidad de Costa Rica: un análisis multinivel. Revista electrónica de investigación y evaluación educativa, 13(2), 215-234.

Pereyra, M., Oros, L., Sicalo, P., y Robles, A. (2017). La familia que soñé. Buenos Aires: Asociación Casa Editora Sudamericana.

Raya, A. (2009). Estudio sobre los estilos educativos parentales y su relación con los trastornos de conducta en la infancia. [Tesis Doctoral]. Universidad de Córdoba.

Sánchez, Y. (2001). Vygotski, Piaget y Freud: A propósito de la socialización. Enunciación, 6(1), 29-34.

Solano, L. (2015). Rendimiento académico de los estudiantes de secundaria obligatoria y su relación con las aptitudes mentales y las actitudes ante el estudio. [Tesis]. Universidad Nacional de Educación a Distancia (UNED).

Torío, S., Peña, J., y Rodríguez, M. (2008). Estilos educativos parentales: revisión bibliográfica y reformulación teórica. Teoría de la Educación, 20, 151-178.

Valdés, Á. (2007). Familia y desarrollo: intervenciones en terapia familiar. México: Editorial El Manual Moderno. 